\title{
The Long-term Outcomes of Endoscopic Stenting as a Bridge to Elective Surgery in Patients with Colorectal Cancer Obstruction as Compared with Emergency Surgery
}

\author{
Yu-Jin Choi, M.D., Jin Kim, M.D., Han-Deok Kwak, M.D., Dong-Woo Kang, M.D., Se-Jin Baek, M.D., Jung-Myun Kwak, M.D.,
} Seon-Hahn Kim, M.D.

Department of Surgery, Korea University College of Medicine, Seoul, Korea

\begin{abstract}
Purpose: The aim of this study was to compare the short- and long-term outcomes between stent placement as a bridge to surgery and emergency surgery for obstructive colon cancer.

Methods: Patients who underwent surgery for left colon cancer and rectal cancer with total obstruction from September 2006 to October 2014 were enrolled. Data for the stent placement and emergency surgery groups were compared.

Results: Of the 67 patients with total obstruction, 53 patients were treated with stent placement and 14 patients were treated with emergency surgery. Significant differences were observed for surgical approach, type of operation, and combined resection. Use of minimally invasive surgery (MIS) was higher ( 88.6 vs. $42.9 \%, p<0.001)$ in the stent placement (SP) group, and combined resection (5.9 vs. $37.5 \%, \mathrm{p}\langle 0.001$ ) was higher in the emergency surgery (EM) group. In the SP group, resection and anastomosis accounted for the largest proportion (92.5\%) and in the EM group, Hartmann's procedure was most common $(57.1 \%)(p<0.001)$. There were no significant differences in other operative outcomes or in postoperative courses. Five-year overall survival was 96.0 and $77.8 \%(p=0.311)$ in the SP and EM groups, respectively. Five-year disease-free survival for local recurrence in the SP and EM groups was 90.0 and $88.9 \%$ ( $p=0.904)$.

Conclusion: Stent placement as a bridge to surgery can be performed safely and represents an alternative to emergency surgery with good short-term results. Stent placement as a bridge to surgery is also comparable to emergency surgery in long-term outcomes.
\end{abstract}

Keywords: Self-expanding metallic stent (SEMS), Colorectal neoplasm, Obstruction, Local recurrence

This is an Open Access article distributed under the terms of the Creative Commons Attribution Non-Commercial License (http:// creativecommons.org/licenses/by-nc/4.0/) which permits unrestricted non-commercial use, distribution, and reproduction in any medium, provided the original work is properly cited.
Received August 31, 2015

Revised October 1, 2015

Accepted October 13, 2015

Corresponding author

Jin Kim

Department of Surgery, Korea

University Anam Hospital, Inchonro 73, Seongbuk-gu, Seoul 02841,

Korea

Tel: +82-2-920-5346

Fax: +82-2-928-1631

E-mail: mrgs@korea.ac.kr
Copyright $@ 2015$ The Journal of Minimally Invasive Surgery. All rights reserved.

\section{INTRODUCTION}

According to statistics recently announced by the Division of Cancer Registration and Surveillance of the National Cancer Center, the incidence of colorectal cancer is increasing in Korea. ${ }^{1}$ Fifteen to twenty percent of colorectal cancer patients have symptoms of intestinal obstruction symptom at the time of diagnosis. ${ }^{2}$ Intestinal obstruction occurs in 7 to $30 \%$ of patients with tumors located in the distal colon of splenic flexure. ${ }^{3}$ Emergency surgical resection is often necessary in patients with obstructive colorectal cancer which has an increased risk of anastomosis leakage due to dilatation 
of proximal colon, ileus, and edema. Emergency surgery also requires stoma formation, and is more likely to need a twostep operation. ${ }^{4,5}$

Since the use of metal stenting was first reported by Dohmoto et al. in 1991, stent placement has facilitated the transition from emergency surgery to elective surgery by decompressing the malignant colorectal obstruction. ${ }^{6-9}$ Stenting as a bridge to surgery makes it possible to avoid stoma formation, allowing the possibility of primary anastomosis. However, stent-related complications are possible including stent dislodgement, bleeding, and perforation. There are also concerns about local cancer recurrence attributable to stenting because of subclinical perforation..$^{10-13}$

Stent placement is set as a standard procedure for malignant obstruction in many tertiary referral hospitals including our institution. This study aimed to investigate the safety, efficacy, and oncologic results for stent placement as a bridge to surgery for left colon and rectal cancer obstruction.

\section{MATERIALS AND METHODS}

The hospital records of all patients who had surgery for malignant colorectal cancer from September, 2006 to October,
2014, at Korea University Anam Hospital were retrospectively reviewed. A total of 2,552 patients had surgery for malignant colorectal cancer. Of these patients, those who had been treated for left colon cancer and rectal cancer with total obstruction were enrolled. The left colon was defined as between the distal transverse colon and rectum. Colorectal cancer total obstruction was defined as intestinal obstruction confirmed through colonoscopy and computed tomography (CT) imaging.

The decision for stent placement was collaboratively made by surgeons and gastroenterologists at our hospital. Emergency surgery was performed in cases of bleeding, perforation with panperitonitis, severe pain suggestive of intestinal ischemia, mid to low rectal tumor location, and for cases of a large fixed mass with a high risk of impending perforation with stenting. Stents were inserted through an endoscope. Self-expandable metallic uncovered stents (M.I. Tech, Gyeonggi-do, Korea) were used with sizes ranging from 20 22 $\mathrm{mm}$ in diameter and 80 140 $\mathrm{mm}$ in length. All stents were placed under endoscopic and fluoroscopic guidance. Technical stent success was defined as successful deployment with fluoroscopic confirmation. The duration from stent placement to elective surgery was established as 7 10 days in our institution.

All data were prospectively collected in a database and

Table 1. Patient demographics and tumor characteristics

\begin{tabular}{|c|c|c|c|}
\hline & $S P(n=53)$ & $\mathrm{EM}(n=14)$ & $p$ \\
\hline Age, mean $\pm S D$ & $66.3 \pm 11.5$ & $65.4 \pm 14.3$ & 0.819 \\
\hline Sex & & & 0.169 \\
\hline Male & $33(62.3 \%)$ & $6(42.9 \%)$ & \\
\hline Female & $20(37.7 \%)$ & $77(34.1 \%)$ & \\
\hline BMI $\left(\mathrm{kg} / \mathrm{m}^{2}\right)$, mean $\pm S D$ & $22.4 \pm 3.0$ & $22.3 \pm 2.3$ & 0.819 \\
\hline Preoperative CEA (ng/ml), mean \pm SD & $75.0 \pm 205.02$ & $13.5 \pm 24.02$ & 0.351 \\
\hline Tumor location & & & 0.767 \\
\hline Descending & $6(11.3 \%)$ & $1(7.1 \%)$ & \\
\hline Sigmoid & $36(67.9 \%)$ & $14(71.4 \%)$ & \\
\hline Rectum & $11(20.8 \%)$ & $3(21.4 \%)$ & \\
\hline Cause of emergency surgery & NA & & NA \\
\hline Perforation & & $5(35.7 \%)$ & \\
\hline Palpable mass on abdominal P/E & & $4(28.6 \%)$ & \\
\hline Severe abdomen pain & & $3(21.4 \%)$ & \\
\hline Mid to low rectal tumor location & & $1(7.1 \%)$ & \\
\hline Bleeding & & $1(7.1 \%)$ & \\
\hline
\end{tabular}

$\mathrm{SP}=$ stent placement group; $\mathrm{EM}=$ emergency operation group; $\mathrm{SD}=$ standard deviation; $\mathrm{BMI}=$ body mass index; $\mathrm{CEA}=$ carcino-embryonic antigen; $N A=$ not applicable; $P / E$ = physical exam. 
analyzed under the approval of the Institutional Review Board in Korea University Anam Hospital. Patient demographics, tumor characteristics, operative and postoperative outcomes and 5-year survivals were compared between patients who had stent placement and those who underwent emergency surgery. SPSS version 20.0 (IBM, Armonk, NY, USA) was used, and the t-test and chi-square test were used for continuous and categorical variables, respectively. Survival analysis was conducted for patients except stage IV cancer patients using Kaplan-Meier analysis with log-rank test. $p$ value less than 0.05 was considered statistically significant.

\section{RESULTS}

A total of 67 patients with left colon cancer and rectal cancer with obstruction were enrolled in our study. Stent placement was performed in 53 patients as a bridge to surgery. Stents effectively decompressed the proximal bowel in all patients, and there were no post-stent placement events such as bleeding or perforation. Fourteen patients received emergency surgery for various reasons (Table 1). Between the two groups, no significant differences were observed in age, sex, body mass index (BMI), preoperative carcino-embryonic antigen (CEA), tumor location. TNM stage, or tumor size.

The operative outcomes are outlined in Table 2. When comparing operative outcomes, there were significant differences in surgical approach, type of operation, and combined resection. Minimally invasive surgery (MIS) (88.6 vs. $42.9 \%$, $p<0.001)$ was higher in the stent placement (SP) as a bridge to surgery group, and combined resection (5.9 vs. $37.5 \%$, $p<0.001)$ was lower in the SP group. With regard to combined resection, four patients were treated due to invasion of pelvic organs, one patient had a gallbladder stone, and one patient had a pheochromocytoma of the adrenal grand. In the SP group, resection with anastomosis was performed in nearly all patients (92.5\%) while in the emergency operation (EM) group,

Table 2. Operative outcomes

\begin{tabular}{|c|c|c|c|}
\hline & $S P(n=53)$ & EM $(n=14)$ & $p$ \\
\hline Surgical approach & & & $<0.001$ \\
\hline MIS (Laparoscopic / Robot) & $47(88.6 \%)$ & $6(42.9 \%)$ & \\
\hline Open & $3(5.7 \%)$ & $8(57.1 \%)$ & \\
\hline Conversion & $3(5.7 \%)$ & 0 & \\
\hline Type of operation & & & $<0.001$ \\
\hline Resection and anastomosis & $49(92.5 \%)$ & $5(35.7 \%)$ & \\
\hline Left hemicolectomy & $6(11.3 \%)$ & $1(7.1 \%)$ & \\
\hline Anterior resection & $26(49.1 \%)$ & $2(14.3 \%)$ & \\
\hline Low anterior resection & $17(32.1 \%)$ & $1(7.1 \%)$ & \\
\hline Total abdominal colectomy & $0(0.0 \%)$ & $1(7.1 \%)$ & \\
\hline Abdominoperineal resection & $0(0.0 \%)$ & $1(7.1 \%)$ & \\
\hline Hartmann's procedure & $4(7.5 \%)$ & $8(57.1 \%)$ & \\
\hline Diverting loop ileostomy* & $9(18.4 \%)$ & $2(40.0 \%)$ & 0.194 \\
\hline Combined resection & $3(5.9 \%)$ & $3(37.5 \%)$ & 0.006 \\
\hline Salphingo-0ophorectomy & $2(3.8 \%)$ & $1(12.5 \%)$ & \\
\hline Hysterectomy & $0(0.0 \%)$ & $1(12.5 \%)$ & \\
\hline Cholecystectomy & $0(0.0 \%)$ & $1(12.5 \%)$ & \\
\hline Adrenalectomy & $1(1.9 \%)$ & $0(0.0 \%)$ & \\
\hline Operation time (min), mean $\pm S D$ & $219.2 \pm 95.3$ & $218.6 \pm 65.7$ & 0.973 \\
\hline Blood loss (ml), mean \pm SD & $30.2 \pm 152.6$ & $635.7 \pm 649.9$ & 0.053 \\
\hline
\end{tabular}

$\mathrm{SP}=$ stent placement group; $\mathrm{EM}=$ emergency operation group; $\mathrm{SD}=$ standard deviation; $\mathrm{MIS}=$ minimal invasive surgery. ${ }^{*}$ Excluded abdominoperineal resection and Hartman's procedure. 
Table 3. Histopathologic results

\begin{tabular}{|c|c|c|c|}
\hline & $S P(n=53)$ & $\mathrm{EM}(n=14)$ & $p$ \\
\hline Differentiation & & & 0.726 \\
\hline Well & $7(13.2)$ & $4(28.6)$ & \\
\hline Moderate & $45(84.9)$ & $8(57.1)$ & \\
\hline Poor & $1(1.9)$ & $2(14.3)$ & \\
\hline Tumor size (cm), mean \pm SD & $6.7 \pm 2.5$ & $5.9 \pm 1.9$ & 0.719 \\
\hline T stage & & & 0.872 \\
\hline $\mathrm{T} 2$ & $1(1.9 \%)$ & $0(0.0 \%)$ & \\
\hline T3 & $45(84.9 \%)$ & $12(85.7 \%)$ & \\
\hline $\mathrm{T} 4$ & $7(13.2 \%)$ & $2(14.3 \%)$ & \\
\hline N stage & & & 0.850 \\
\hline NO & $22(41.5 \%)$ & $7(50.0 \%)$ & \\
\hline N1 & $22(41.5 \%)$ & $5(35.7 \%)$ & \\
\hline N2 & $9(17.0 \%)$ & $2(14.3 \%)$ & \\
\hline M stage & & & 0.520 \\
\hline MO & $29(54.7 \%)$ & $9(64.3 \%)$ & \\
\hline M1 & $24(45.3 \%)$ & $5(35.7 \%)$ & \\
\hline TNM stage & & & 0.664 \\
\hline$\|$ & $16(30.2 \%)$ & $6(42.9 \%)$ & \\
\hline$\|$ & $13(24.5 \%)$ & $3(21.4 \%)$ & \\
\hline IV & $24(45.3 \%)$ & $5(35.7 \%)$ & \\
\hline $\begin{array}{l}\text { Distal resection margin }(\mathrm{cm}) \text {, } \\
\text { mean } \pm S D\end{array}$ & $6.4 \pm 5.0$ & $8.6 \pm 7.9$ & 0.073 \\
\hline
\end{tabular}

$S P=$ stent placement group; $E M=$ emergency operation group; $S D=$ standard deviation.

Hartmann's procedure was most commonly performed (57.1\%) $(p<0.001)$. Only $50 \%$ of patients who underwent Hartmann's procedure could be received the reversal operation later (the SP group vs. the EM group, 75 vs. $37.5 \%$, respectively; $p=0.221$ ). The two groups did not show a difference in diverting loop ileostomy, operative time, or the amount of blood loss. All diverting loop ileostomy were able to be repaired later.

No significant differences were demonstrated in histopathologic results between the two groups (Table 3). Postoperative courses and adjuvant treatment are shown in Table 4. There were no significant differences between the two groups with regard to postoperative hospital stay (16.7 vs. 14.9 days, $p=0.103$ ), days to flatus ( 2.2 vs. 2.4 days, $p=0.809$ ), days to resumption of diet (3.3 vs. 5.4 days, $p=0.102$ ), postoperative complications (17.0 vs. $7.1 \%, p=0.870$ ), or the types of adjuvant
Table 4. Postoperative courses and adjuvant treatment

\begin{tabular}{lccc} 
& SP $(\mathbf{n}=53)$ & EM $(\mathbf{n}=14)$ & $p$ \\
\hline $\begin{array}{l}\text { Postoperative hospital stay, } \\
\text { median (ranges) }\end{array}$ & $16.7(5 \sim 88)$ & $14.9(6 \sim 190)$ & 0.103 \\
Days to flatus, median (ranges) & $2.2(0 \sim 7)$ & $2.4(2 \sim 4)$ & 0.809 \\
Days to diet, median (ranges) & $3.3(1 \sim 15)$ & $5.4(2 \sim 19)$ & 0.102 \\
Postoperative Complications & & & 0.870 \\
Wound infection & $5(9.4 \%)$ & $1(7.1 \%)$ & \\
Anastomotic leakage & $2(3.8 \%)$ & 0 & \\
Anastomotic ischemia & $1(1.9 \%)$ & 0 & \\
Small bowel perforation & $1(1.9 \%)$ & 0 & \\
Adjuvant chemotherapy & $38(71.7 \%)$ & $11(78.6 \%)$ & 0.901 \\
$5-F U$ & $1(2.6 \%)$ & 0 & \\
FOLFOX & $30(56.6 \%)$ & $9(64.3 \%)$ & \\
FOLFIRI & $7(18.4 \%)$ & $2(18.2 \%)$ &
\end{tabular}

FU = fluorouracil; FOLFOX = oxaliplatin-based chemotherapy; FOLFIRI = irinotecan-based chemotherapy.

chemotherapy $(p=0.901)$.

We compared long-term outcomes for patients except for stage IV patients. Five-year overall survival (OS) rates were 96.0 and $77.8 \%$ ( $p=0.311)$ in the SP and EM groups respectively (Fig. 1). The median survival time was 65.6 months in the SP group and 69.2 months in the EM group. Five-year OS rates were $100.0 \%$ in the SP group and $75.0 \%$ in the EM group for stage II $(p=0.448)$, and $96.0 \%$ in the SP group and $77.8 \%$ in the EM group for stage III $(p=0.560)$. Five-year disease-free survival (DFS) rates for local recurrence in the SP and EM groups were 90.0 and $88.9 \%(p=0.904)$. Five-year DFS rates for local recurrence were $83.3 \%$ in the SP group and $100.0 \%$ in the EM group for stage II $(p=0.564)$, and were $100.0 \%$ in the SP group and $75.0 \%$ in the EM group for stage III $(p=0.750)$ (Fig. 2) And, five-year DFS rates for systemic recurrence in the SP and EM groups were 92.9 and $66.7 \%(p=0.219)$. Fiveyear DFS rates for systemic recurrence were $93.4 \%$ in the SP group and $80 \%$ in the EM group for stage II $(p=0.469)$, and were $83.3 \%$ in the SP group and $58.3 \%$ in the EM group for stage III ( $p=0.922)$ (Fig. 3).

\section{DISCUSSION}

In our study, we found the minimally invasive surgery was performed actively in the SP group and the percentage of resection and anastomosis was higher compared with the use of Hartmann's operation in the SP group. We observed comparable postoperative progress and long- term results 
A

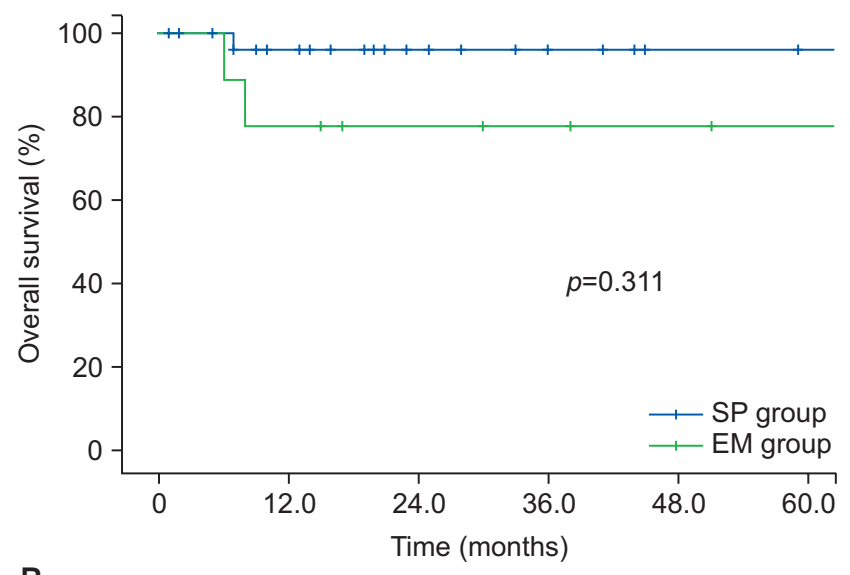

B

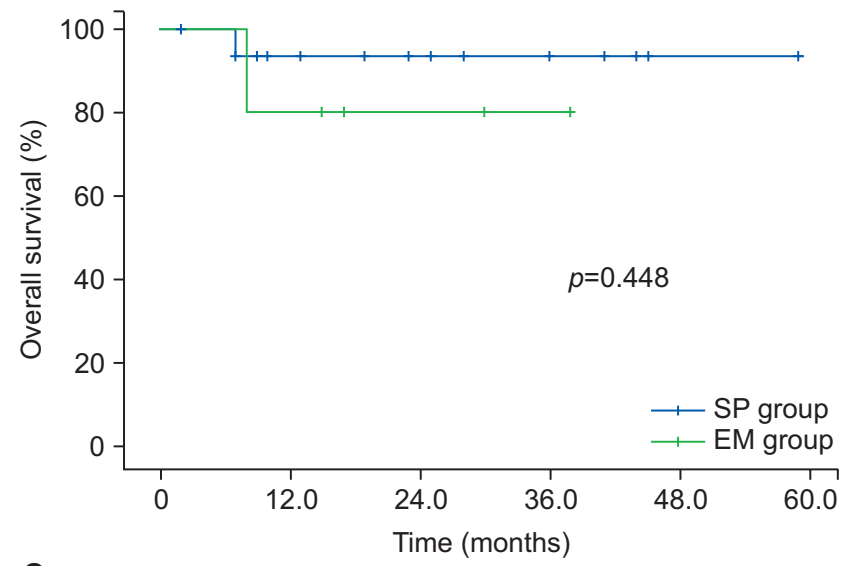

C

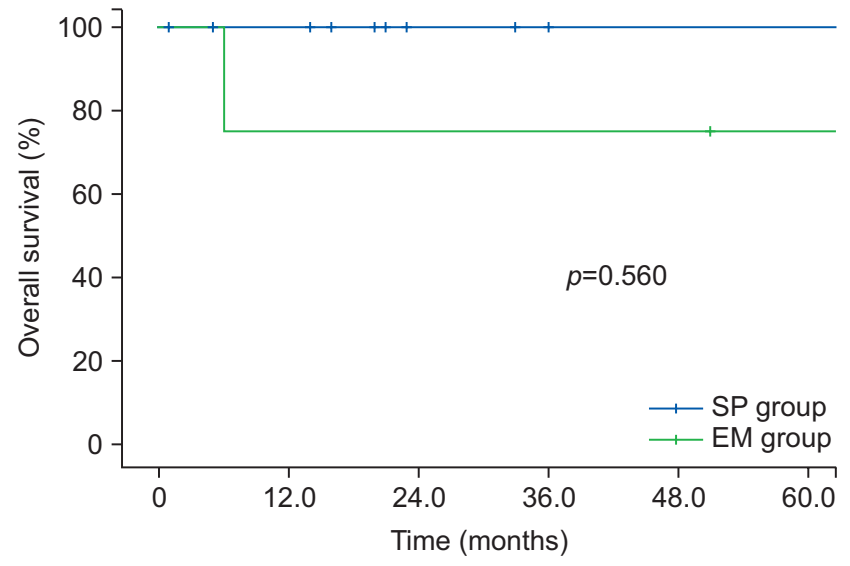

Fig. 1. Comparison of 5-year overall survival in patients with malignant colonic obstruction treated with stent as a bridge to surgery or emergency surgery only. (A) All stage, (B) Stage II, (C) Stage III. SP = stent placement group; $\mathrm{EM}=$ emergency operation group.

between the SP and EM groups.

Hartmann's operation is generally performed as a temporary method to stabilize left-sided colonic emergencies including colonic obstruction, perforation, bleeding or anastomotic
A

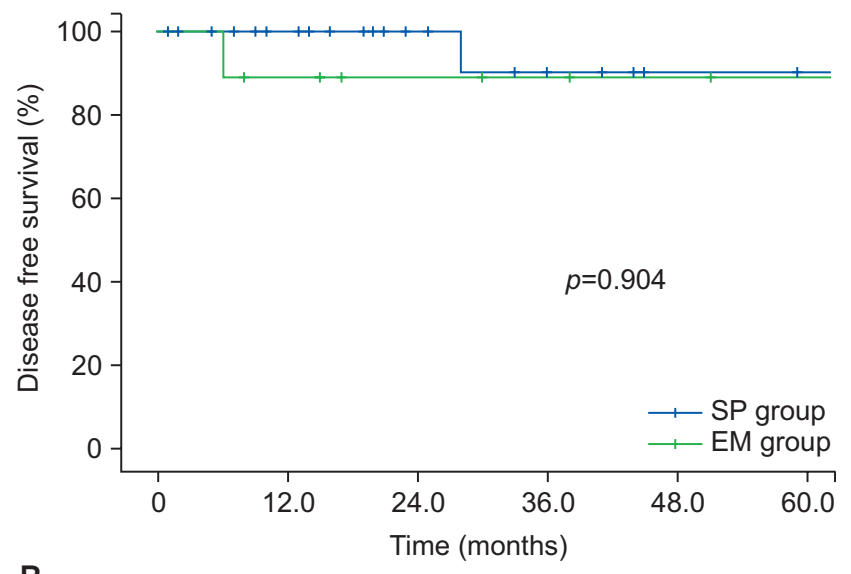

B

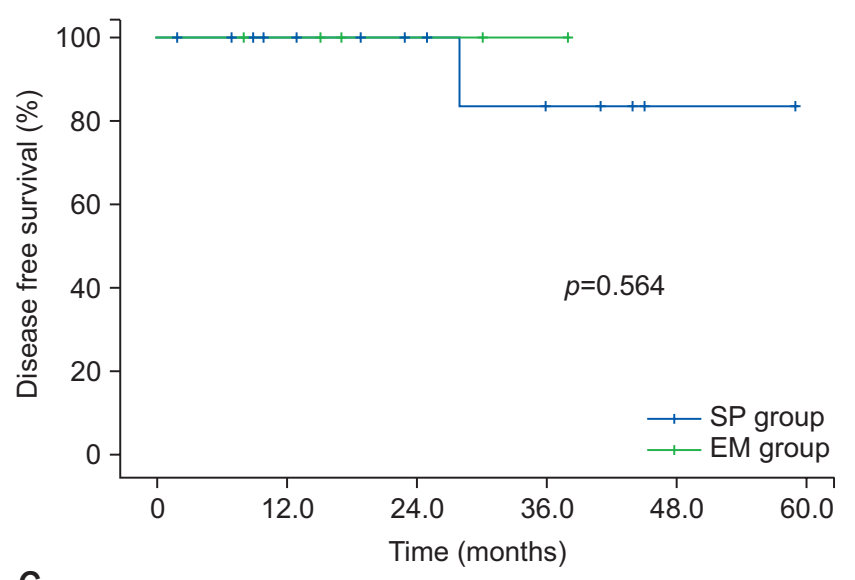

C

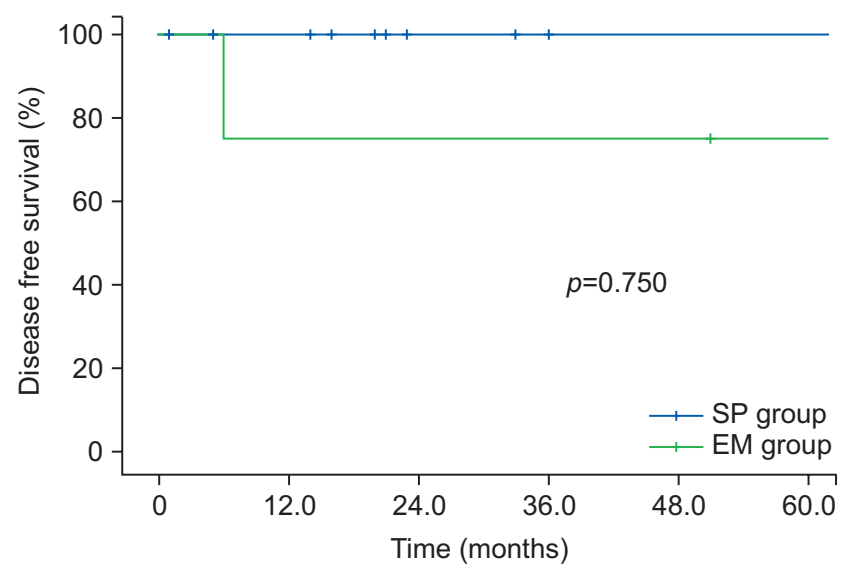

Fig. 2. Comparison of 5-year disease free survival for local recurrence in patients with malignant colonic obstruction treated with stent as a bridge to surgery or emergency surgery only. (A) All stage, (B) Stage II, (C) Stage III. SP = stent placement group; $\mathrm{EM}=$ emergency operation group.

leakage that are not appropriate for primary anastomosis. The subsequent restoration of bowel continuity is advisable for better quality of life, but it may be technically challenging for 
A

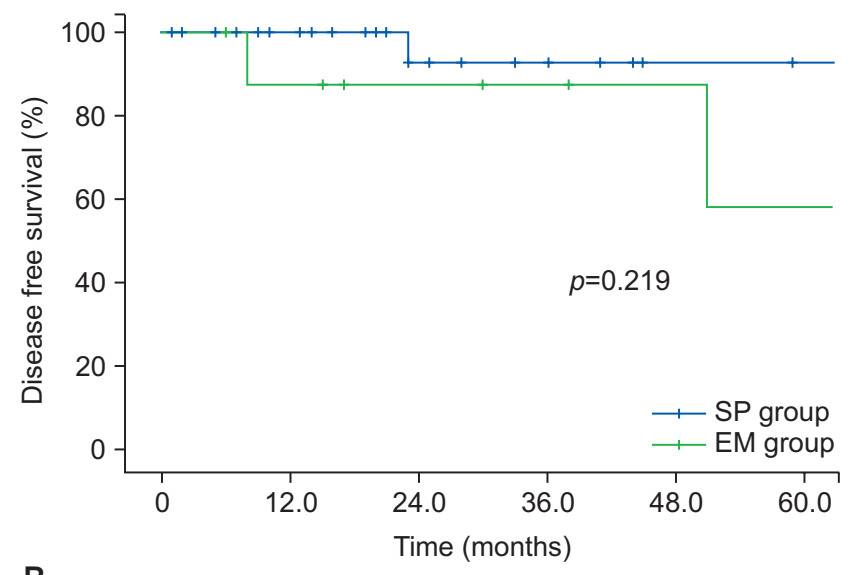

B

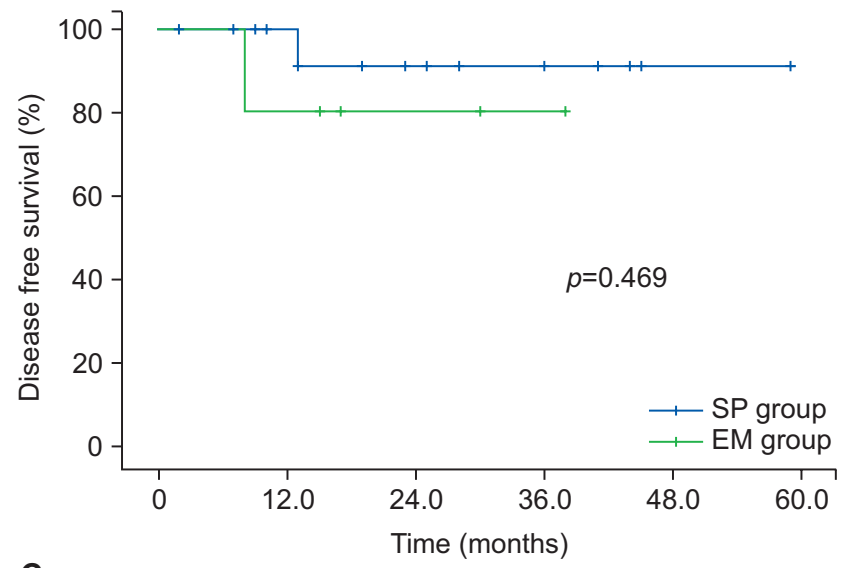

C

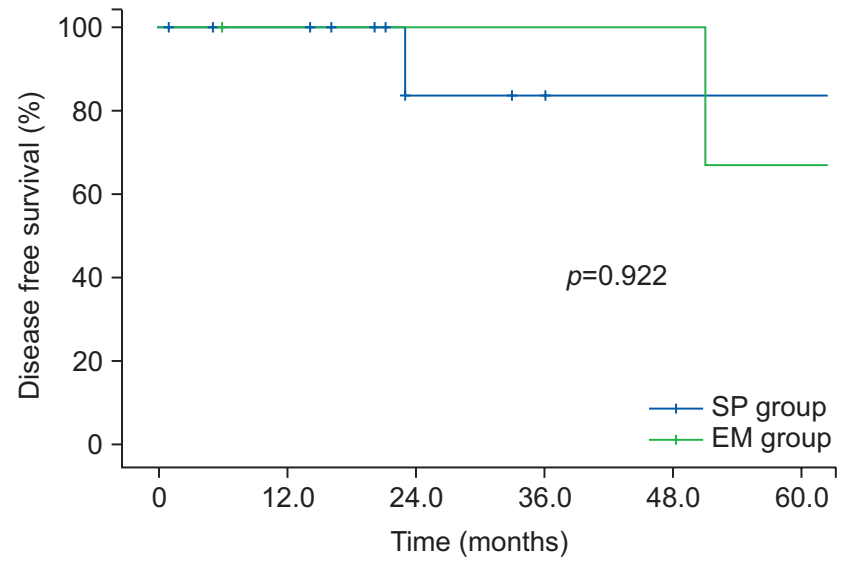

Fig. 3. Comparison of 5-year disease free survival for systemic recurrence in patients with malignant colonic obstruction treated with stent as a bridge to surgery or emergency surgery only. (A) All stage, (B) Stage II, (C) Stage III. SP = stent placement group; $\mathrm{EM}=$ emergency operation group.

surgeons. In addition, the reversal of Hartmann's operation has considerable morbidity and mortality including postoperative leakage, stricture, bleeding, and wound infections. For this reason, re-anastomosis is performed in only 55 to $60 \%$ of patients who receive Hartmann's operation. ${ }^{14-18}$ Our study also showed the rate of Hartmann's reversal operation was $75 \%$ in the SP group, 37.5\% in the EM group, and 50\% in total. Therefore, the lower rate of Hartmann's operation in the stent placement patients may positively influence long-term quality of life, as seen in our study.

Most of the SP group patients were able to have minimally invasive surgery, such as laparoscopic or robotic surgery. The availability of laparoscopic surgery may be affected by various factors, and it is difficult to perform laparoscopic surgery in cases of severe ileus of the proximal bowel due to obstruction of the distal colon. For these reasons, the proportion of patients who underwent surgery using MIS was likely higher in the SP group because of effective proximal bowel decompression by stenting. As it is well known, laparoscopic surgery has great something compared to open surgery including reduced postoperative pain, early recovery, faster resumption of diet, and improved cosmesis. ${ }^{19,20}$ The SP group patients were able to continue these advantages as well. In our study, there was one adrenalectomy as a combined operation due to pheochromocytoma. Pheochromocytoma surgery is high risk and requires intensive preparation before surgery related to hormone secretion. In these patients, the risk of emergency surgery is much higher than in well-planned, elective surgery. In this case, effective stent placement allowed elective colectomy with adrenalectomy to be performed safely. If the patient has poor general condition or a critical co-morbidity and is at risk of deteriorate condition under general anesthesia such as thyrotoxic crisis, called thyroid storm which can cause a hypertensive crisis, stenting may allow a period for recovery and improvement before elective surgery to reduce morbidity and mortality compared with emergency surgery. ${ }^{21,22}$

Contrary to our expectations, there were no significant differences in postoperative outcomes between the SP and EM groups. We apply the enhanced recovery after surgery (ERAS) protocol to patients undergoing laparoscopic and open surgery both in our institution. ${ }^{23,24}$ The shared protocol may explain why the EM group, which included more patients who underwent open surgery, did not show inferior results in terms of postoperative diet resumption or hospital stay compared with the SP group.

Recently, reports have raised the possibility that stent insertion increases local cancer recurrence due to subclinical perforation during implantation. Concern for cancer cells causing metastasis along peripheral blood vessels with worsened survival has been suggested. Kim et al. ${ }^{25}$ reported that SEMS insertion had an adverse effect on the five-year overall survival rate (SEMS group vs. emergency surgery group, 38.4 vs. $65.6 \%$, respectively; $p=0.025$ ) and the fiveyear disease-free survival rate (SEMS group vs. emergency 
surgery group, 48.3 vs. $75.5 \%$, respectively; $p=0.024$ ). On the other hand, long-term outcomes were not significantly different between the two groups in our study. Five-year overall survival was 96.0 and $77.8 \%$ ( $p=0.311)$ in the SP group and EM groups respectively. Five-year disease-free survival for local recurrence in the SP and EM groups were 90.0 and $88.9 \%(p=0.904)$. Similarly, Saida et al. ${ }^{26}$ reported that longterm outcomes did not significantly differ and the five-year overall survival rate was $40 \%$ in the expandable metallic stent group and $44 \%$ in the emergency operation group. Choi et al. ${ }^{27}$ also showed no significant difference in long-term prognosis between two groups in either the five-year disease-free survival for recurrence (SEMS group vs. emergency surgery group, 79.6 vs. $70.2 \%, p=0.218$ ) or the five-year overall survival rate (SEMS group vs. emergency surgery group, 97.8 vs. $94.3 \%$, $p=0.469$ ).

To date, no study has been published that definitively shows that either approach is worse than the other considering the institutional and technical bias. That being said, stent placement for malignant obstruction has found a solid home for the surgical treatment option of malignant obstruction so far. However, there remains debate regarding the effect of stent insertion for long-term oncologic outcomes, and future studies with long-term follow-up result including CReST (ColoRectal Stenting Trial) is expected.

Our study has a few limitations. First, our study is a retrospective analysis and suffers from selection bias. We did not compare the results from randomized patients following stent insertion or emergency surgery, and instead reviewed the results of patients who were not able to undergo stent placement and underwent emergency surgery. In addition, the decision and ability to place stents is somewhat subjective and can differ across institutions. The success rate of stent placement in our study was 100\%, and this is higher than the 86 98\% generally reported. ${ }^{28,29}$ This suggests that there are well-trained endoscopists at our hospital but also could reflect that stent insertion as a bridge to surgery was more narrowly considered as an option. Finally, this study reflects a small amount of single-center data and a relatively small patient group. That is due to the definition for total obstruction in the enrolled patients.

In conclusion, colonic stenting can be used safely as a bridge to elective surgery as an alternative to emergency surgery in patients with left colon and rectal cancer obstruction. Stent placement as a bridge to surgery demonstrated comparable results with emergency surgery with regard to long-term oncologic outcomes.

\section{ACKNOWLEDGEMENTS}

Choi YJ mainly contributed to this paper, Kim J edited, Kwak HD, Kang DW, Baek SJ, Kwak J and Kim SH reviewed generally.

\section{REFERENCES}

1) Cancer statics in Korea. Division of cancer registration \& surveillance of national cancer center 2012.

2) Fan YB, Cheng YS, Chen NW, Xu HM, Yang Z, Wang Y, Huang YY, Zheng Q. Clinical application of self-expanding metallic stent in the management of acute left-sided colorectal malignant obstruction. World J Gastroenterol 2006;12:755-759.

3) A Maffioli AB, G Manes, D Cavallo, S Callioni, PG Danell. Colonic stenting in the management of malignant intestinal obstruction in elderly patients. BMC Surg 2013.

4) Villar JM, Martinez AP, Villegas MT, Muffak K, Mansilla A, Garrote D, Ferron JA. Surgical options for malignant left-sided colonic obstruction. Surg Today 2005;35:275-281.

5) Zorcolo L, Covotta L, Carlomagno N, Bartolo DC. Safety of primary anastomosis in emergency colo-rectal surgery. Colorectal Dis 2003;5:262-269.

6) Dohmoto M, Rupp KD, Hohlbach G. Endoscopically-implanted prosthesis in rectal carcinoma. Dtsch Med Wochenschr 1990;115: 915.

7) Geraghty J, Sarkar S, Cox T, Lal S, Willert R, Ramesh J, Bodger K, Carlson GL. Management of large bowel obstruction with self-expanding metal stents. A multicentre retrospective study of factors determining outcome. Colorectal Dis 2014;16:476-483.

8) Jimenez-Perez J, Casellas J, Garcia-Cano J, Vandervoort J, Garcia-Escribano OR, Barcenilla J, Delgado AA, Goldberg P, Gonzalez-Huix F, Vazquez-Astray E, Meisner S. Colonic stenting as a bridge to surgery in malignant large-bowel obstruction: a report from two large multinational registries. Am J Gastroenterol 2011;106:2174-2180.

9) Jury G, Amieva L, Dolan M, Fagalde RL, Naiderman D, Pastorino M, Jury R. Neoplasic colonic obstruction: resolution with selfexpanding metallic stents. Acta Gastroenterol Latinoam 2014;44: 33-38.

10) Kavanagh DO, Nolan B, Judge C, Hyland JM, Mulcahy HE, O'Connell PR, Winter DC, Doherty GA. A comparative study of short- and medium-term outcomes comparing emergent surgery and stenting as a bridge to surgery in patients with acute malignant colonic obstruction. Dis Colon Rectum 2013;56:433-440.

11) Maruthachalam K, Lash GE, Shenton BK, Horgan AF. Tumour cell dissemination following endoscopic stent insertion. Br J Surg 2007;94:1151-1154.

12) Malgras B, Brulle L, Lo Dico R, El Marjou F, Robine S, Therwath A, Pocard M. Insertion of a stent in obstructive colon cancer can 
induce a metastatic process in an experimental murine model. Ann Surg Oncol 2015.

13) Sloothaak DA, van den Berg MW, Dijkgraaf MG, Fockens P, Tanis PJ, van Hooft JE, Bemelman WA, collaborative Dutch Stent-In study g. Oncological outcome of malignant colonic obstruction in the Dutch Stent-In 2 trial. Br J Surg 2014;101:17511757.

14) Parkin E, Khurshid M, Ravi S, Linn T. Surgical access through the stoma for laparoscopic reversal of Hartmann procedures. Surg Laparosc Endosc Percutan Tech 2013;23:41-44.

15) Lin FL, Boutros M, Da Silva GM, Weiss EG, Lu XR, Wexner SD. Hartmann reversal: obesity adversely impacts outcome. Dis Colon Rectum 2013;56:83-90.

16) Van de Wall BJ, Draaisma WA, Schouten ES, Broeders IA, Consten EC. Conventional and laparoscopic reversal of the Hartmann procedure: a review of literature. J Gastrointest Surg 2010;14:743-752.

17) Zimmermann M, Hoffmann M, Laubert $T$, Meyer KF, Jungbluth T, Roblick UJ, Bruch HP, Schloricke E. Laparoscopic versus open reversal of a Hartmann procedure: a single-center study. World J Surg 2014;38:2145-2152.

18) Aydin C, Olmez A, Isik S, Sumer F, Kayaalp C. Reversal of the Hartmann procedure through only a stomal orifice. Am Surg 2011; 77:694-696.

19) Cheung HY, Chung CC, Tsang WW, Wong JC, Yau KK, Li MK. Endolaparoscopic approach vs conventional open surgery in the treatment of obstructing left-sided colon cancer: a randomized controlled trial. Arch Surg 2009;144:1127-1132.

20) Yamamoto S, Inomata M, Katayama H, Mizusawa J, Etoh T, Konishi F, Sugihara K, Watanabe M, Moriya Y, Kitano S, Japan Clinical Oncology Group Colorectal Cancer Study G. Short-term surgical outcomes from a randomized controlled trial to evaluate laparoscopic and open D3 dissection for stage II/III colon cancer: Japan Clinical Oncology Group Study JCOG 0404. Ann Surg 2014; 260:23-30.

21) Zambouri A. Preoperative evaluation and preparation for anesthesia and surgery. Hippokratia 2007;11:13-21.

22) Yamauchi H, Sobue K. Anesthesia preoperative preparation of muscular dystrophy. Masui 2010;59:1093-1095.

23) Shida $D$, Tagawa $K$, Inada $K$, Nasu $K$, Seyama $Y$, Maeshiro $T$, Miyamoto S, Inoue S, Umekita N. Enhanced recovery after surgery (ERAS) protocols for colorectal cancer in Japan. BMC Surg 2015;15:90.

24) Spanjersberg WR, van Sambeeck JD, Bremers A, Rosman C, van Laarhoven CJ. Systematic review and meta-analysis for laparoscopic versus open colon surgery with or without an ERAS programme. Surg Endosc 2015.

25) Kim JS, Hur H, Min BS, Sohn SK, Cho CH, Kim NK. Oncologic outcomes of self-expanding metallic stent insertion as a bridge to surgery in the management of left-sided colon cancer obstruction: comparison with nonobstructing elective surgery. World J Surg 2009;33:1281-1286.

26) Saida Y, Sumiyama Y, Nagao J, Uramatsu M. Long-term prognosis of preoperative "bridge to surgery" expandable metallic stent insertion for obstructive colorectal cancer: comparison with emergency operation. Dis Colon Rectum 2003;46:S44-S49.

27) Choi JM, Lee C, Han YM, Lee M, Choi YH, Jang DK, Im JP, Kim SG, Kim JS, Jung HC. Long-term oncologic outcomes of endoscopic stenting as a bridge to surgery for malignant colonic obstruction: comparison with emergency surgery. Surg Endosc 2014;28:2649-2655.

28) Fregonese D, Naspetti R, Ferrer S, Gallego J, Costamagna G, Dumas R, Campaioli M, Morante AL, Mambrini P, Meisner S, Repici A, Andreo L, Masci E, Mingo A, Barcenilla J, Petruzziello L. Ultraflex precision colonic stent placement as a bridge to surgery in patients with malignant colon obstruction. Gastrointest Endosc 2008;67:68-73.

29) Won Y, Lee SL, Ku YM, Kim KT, Won HS, An CH. Clinical effect of double coaxial self-expandable metallic stent in management of malignant colon obstruction. Diagn Interv Radiol 2015;21: 167-172. 